\title{
Experimental evaluation of the pure configurational stress assumption in the flow dynamics of entangled polymer melts
}

Rasmussen, Henrik K.; Bejenariu, Anca Gabriela; Hassager, Ole; Auhl, D.

Published in:

Journal of Rheology

Link to article, DOI:

$10.1122 / 1.3496378$

Publication date:

2010

Document Version

Publisher's PDF, also known as Version of record

Link back to DTU Orbit

Citation (APA):

Rasmussen, H. K., Bejenariu, A. G., Hassager, O., \& Auhl, D. (2010). Experimental evaluation of the pure configurational stress assumption in the flow dynamics of entangled polymer melts. Journal of Rheology, 54(6), 1325-1336. https://doi.org/10.1122/1.3496378

\section{General rights}

Copyright and moral rights for the publications made accessible in the public portal are retained by the authors and/or other copyright owners and it is a condition of accessing publications that users recognise and abide by the legal requirements associated with these rights.

- Users may download and print one copy of any publication from the public portal for the purpose of private study or research.

- You may not further distribute the material or use it for any profit-making activity or commercial gain

- You may freely distribute the URL identifying the publication in the public portal 


\title{
Experimental evaluation of the pure configurational stress assumption in the flow dynamics of entangled polymer melts
}

\author{
Henrik Koblitz Rasmussen ${ }^{\text {a) }}$ \\ Department of Mechanical Engineering, Technical University of Denmark, \\ DK-2800 Kgs. Lyngby, Denmark \\ Anca Gabriela Bejenariu and Ole Hassager \\ Department of Chemical and Biochemical Engineering, Technical University of \\ Denmark, DK-2800 Kgs. Lyngby, Denmark \\ Dietmar Auhl ${ }^{\text {b) }}$ \\ IRC in Polymer Science and Technology, University of Leeds, Leeds LS2 9JT, \\ United Kingdom
}

(Received 17 March 2010; final revision received 9 September 2010;

published 11 October 2010)

\section{Synopsis}

A filament stretching rheometer was used for measuring the startup of uni-axial elongational flow followed by reversed bi-axial flow, both with a constant elongational strain rate. A narrow molecular mass distribution linear polyisoprene with a molecular weight of $483 \mathrm{~kg} / \mathrm{mole}$ was subjected to the flow in the non-linear flow regime. This has allowed highly elastic measurements within the limit of pure orientational stress, as the time of the flow was considerably smaller than the Rouse time. A Doi-Edwards [J. Chem. Soc., Faraday Trans. 2 74, 1818-1832 (1978)] type of constitutive model with the assumption of pure configurational stress was accurately able to predict the startup as well as the reversed flow behavior. This confirms that this commonly used theoretical picture for the flow of polymeric liquids is a correct physical principle to apply. (C) 2010 The Society of Rheology. [DOI: 10.1122/1.3496378]

\section{INTRODUCTION}

In the advancement of the understanding of flow dynamics of the entangled polymer systems, a large number of constitutive theories have been suggested during the last 40 years [Doi and Edwards (1978); Marrucci and Grizzuti (1988); Pearson et al. (1999); Mead et al. (1998); McLeish and Larson (1998); Fang et al. (2000); Ianniruberto and Marrucci (2001); Schieber et al. (2003); Likhtman (2005); Wagner et al. (2005, 2008);

\footnotetext{
${ }^{a}$ Author to whom correspondence should be addressed; electronic mail: hkra@mek.dtu.dk

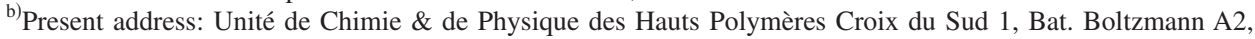
Louvain-la-Neuve B-1348, Belgium.
} 
Khaliullin and Schieber (2009); Rasmussen et al. (2009); Dhole et al. (2009) and others]. Theories all predict fundamentally different flow behaviors. With few exceptions the theories are based on the idea that a given polymer chain is moving by reptation in a tube made up effectively by the surrounding chains [de Gennes (1979)]. The large number of theories is due to the difficulties to obtain accurate and reliable experimental observations in a homogeneous flow of a theoretically ideal system. Its observations may lay the foundation to a more sound theoretical framework. The ideal systems in entangled polymers are monodisperse polymer melts, which may be structurally complex. In shear flow, shearbanding disturbs the homogeneous flow field [Adams and Olmsted (2009)] whereas necking phenomena [Lyhne et al. (2009)] as well as fracturing [Joshi and Denn (2003)] may appear in extension.

The first measurements on the extension of monodisperse melts, including the startup as well as the steady flow, were presented in 2003 [Bach et al. (2003a)]. These data removed the validity of all existing theories for the flow of polymer melts [Marrucci and Ianniruberto (2004)] and inspired new ones to be developed. Since then several new and more dynamical extensional measurements have been published based on the same technique as Bach et al. (2003a). These new extensional measurements include uni-axial extensional flow following by stress relaxation [Nielsen et al. (2008)] or bi-axial reversed flow [Nielsen and Rasmussen (2008)] as well as large amplitude oscillatory elongation [Rasmussen et al. (2008)]. The most recent work is on architecturally complex molecules [Rasmussen et al. (2009)].

Here, in the present study the purpose here is to take a look at the physical basis shared by almost all models based on the concept that a given polymer chain is moving by reptation. The intension is to experimentally evaluate if the pure configurational stress assumption, which is the commonly used theoretical picture for the flow of entangled polymeric liquids, is an accurate physical principle. According to our knowledge, experiments capable of doing this have not been presented before.

\section{CONSTITUTIVE THEORIES}

Constitutive theories having the monodisperse polymer melt as model molecule were pioneered by Doi and Edwards (1978). They constructed a model for the dynamics of highly entangled monodisperse polymer melts within the ideas of de Gennes (1979). While the original Doi and Edwards model [Doi and Edwards (1978)] has many limitations, the basic idea of a confining tube is still utilized extensively in the modeling of entangled polymer systems. In the limit of a purely orientational stress, understood as the time of the flow is considerably smaller than the Rouse time $\tau_{R}$, most of these models appear similar to the Doi and Edwards model. Here we write the model as a memoryweighted time integral over the Doi and Edwards strain tensor strain tensor, in the independent alignment approximation [Doi and Edwards 1978)], as

$$
\sigma_{\mathrm{ij}}=\int_{-\infty}^{t} M\left(t-t^{\prime}\right) 5\left\langle\frac{E_{\mathrm{in}} u_{\mathrm{n}} E_{\mathrm{jm}} u_{\mathrm{m}}}{|\mathbf{E} \cdot \mathbf{u}|^{2}}\right\rangle d t^{\prime},
$$

where the terms $\sigma_{\mathrm{ij}}$ are the integral components of the stress tensor. The angular brackets denote an average over a unit sphere $\langle\cdots\rangle=1 /(4 \pi) \int_{|\mathbf{u}|=1} \cdots d \mathbf{u}$ where a tube segment of unit length and orientation is given by the unit vector $\mathbf{u}$. In the stress free state $\mathbf{u}$ is deformed into $\mathbf{E} \cdot \mathbf{u}$ in the current state. The components of the macroscopic displacement gradient tensor is given by $E_{\mathrm{ij}}\left(\boldsymbol{x}, t, t^{\prime}\right)=\partial x_{\mathrm{i}} / \partial x_{\mathrm{j}}^{\prime}, i=1,2,3$ and $j=1,2,3 .\left(x_{1}^{\prime}, x_{2}^{\prime}, x_{3}^{\prime}\right)$ are the coordinates of a given particle in the stress free reference state (time $t^{\prime}$ ), displaced to 
coordinates $\left(x_{1}, x_{2}, x_{3}\right)$ in the current state (time $\left.t\right)$. The memory function $M\left(t-t^{\prime}\right)$ is related to the relaxation modulus as $M\left(t-t^{\prime}\right)=d G\left(t-t^{\prime}\right) / d t^{\prime}$.

Although experimental findings in extension seem to confirm the theoretical picture of the independent alignment for monodisperse linear melts [Rasmussen et al. (2008)] we include the stress based on the idea of an entangled network with instantaneous chain retraction [Doi and Edwards (1986)] given as

$$
\sigma_{\mathrm{ij}}=\int_{-\infty}^{t} M\left(t-t^{\prime}\right) \frac{15}{4} \frac{1}{\langle|\mathbf{E} \cdot \mathbf{u}|\rangle}\left\langle\frac{E_{\mathrm{in}} u_{\mathrm{n}} E_{\mathrm{jm}} u_{\mathrm{m}}}{|\mathbf{E} \cdot \mathbf{u}|}\right\rangle d t^{\prime} .
$$

Analytical formulas as well as theoretical concepts of the unit sphere integrals can be found in Urakawa et al. (1995). The time integration is performed using a numerical integration scheme.

The only unknown in Eqs. (1) and (2) is the memory function. An understanding and analytical theory of the memory function for monodisperse polymer melt has been presented by Milner and McLeish (1998). Here we will apply the empirical method by Baumgaertel et al. (1990) (BSW), as it gives the most accurate prediction of mechanical spectroscopical data.

Stresses in polymers are assumed to be only of entropic nature. Reversing flow experiments give particular insight into the entropic state of the melt as it mounts the work performed by the polymer. Nielsen and Rasmussen (2008) presented the first and currently the only measurements of well defined reversed elongational flow on a monodisperse linear polystyrene melt, applying uni-axial extensional flow followed by a bi-axial reversed flow. They showed that constitutive models, which predict almost the same flow behavior in the start up of uni-axial extensional flow, behave fundamentally different during the reversed flow.

The same type of measurements as in Nielsen and Rasmussen (2008) are here applied on a monodisperse polyisoprene (PI) melt. The presently used polyisoprene melt contains of about 100 entanglements and a ratio between the reptation and the Rouse time of 200-300, depending on the used definitions, according to Auhl et al. (2008). This allows highly elastic measurements within the limit of pure orientational stress. The present polyisoprene has a molecular weight $M_{w}=483 \mathrm{~kg} /$ mole and a polydispersity $M_{w} / M_{n}$ $=1.03$. $M_{w}$ and $M_{n}$ are weight and mole based average molar masses, respectively. Details, including mechanical spectropical data, on this melt can be found in Auhl et al. (2008). Particular the plateau modulus $G_{N}^{0}$ has been determined to $470 \mathrm{kPa}$. These mechanical spectropical data of the polyisoprene melt are also shown in Fig. 1.

The relaxation modulus was obtained using small amplitude oscillatory shear measurements and fitted [Rasmussen et al. (2000)] with a continuous BSW relaxation spectrum [Baumgaertel et al. (1990)]

$$
\begin{gathered}
G\left(t-t^{\prime}\right)=\int_{0}^{\infty} \frac{H(\tau)}{\tau} e^{\left(-\left(t-t^{\prime}\right) / \tau\right)} d \tau, \\
H(\tau)=n_{e} G_{N}^{0}\left[\left(\frac{\tau}{\tau_{\max }}\right)^{n_{e}}+\left(\frac{\tau}{\tau_{c}}\right)^{-n_{g}}\right] h\left(1-\tau / \tau_{\max }\right)
\end{gathered}
$$

as shown in Fig. 1. $h(x)$ is the Heaviside step function, $n_{e}$ is the slope of the $\left(\log (\omega), \log G^{\prime}\right)$ curve at intermediate frequencies $\omega, n_{g}$ is the slope of $\left(\log (\omega), \log G^{\prime \prime}\right)$ for $\omega \rightarrow \infty$, and $\tau_{c}$ is the crossover relaxation time. The linear viscoelastic parameters are given in Table I where the plateau modulus, $G_{N}^{0}$, is the one from Auhl et al. (2008). 


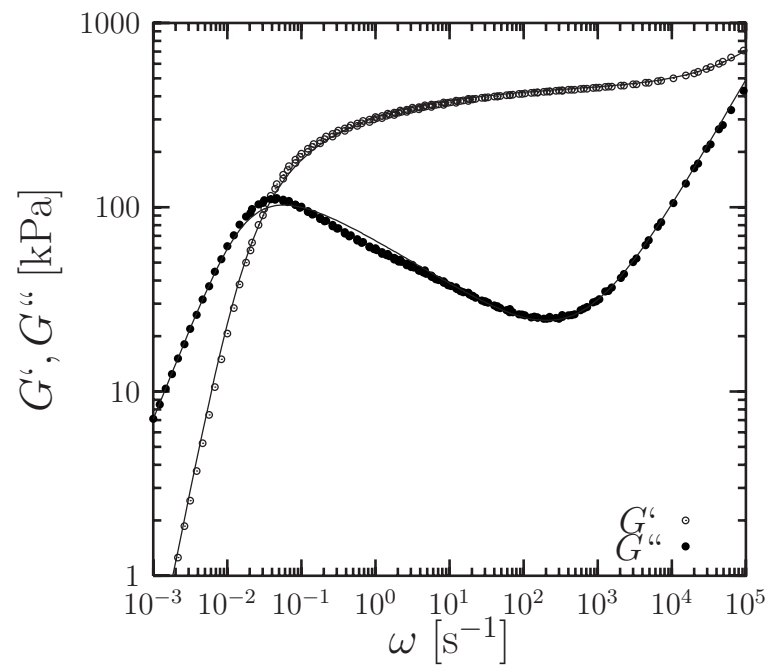

FIG. 1. Measured loss, $G^{\prime \prime}$ (bullets; -) and storage moduli, $G^{\prime}$ (open circles; $\bigcirc$ ) both as a function of the angular frequency, $\omega$, from Auhl et al. (2008) at $23^{\circ} \mathrm{C}$. The solid lines (-) are the least-squares fittings [Rasmussen et al. (2000)] to the BSW model in Eq. (3).

\section{EXTENSIONAL RHEOMETRY}

The extensional experiments are performed using a filament stretching rheometer (FSR) constructed by Bach et al. (2003b). In this FSR, a cylindrical shaped liquid sample, with height $L_{\mathrm{i}}$ and radius $R_{\mathrm{i}}$, is placed between two parallel solid cylinders. The separation of these cylinders will extend the sample. The FSR enables measurements of the strain in the sample exactly at the location of the necking at the mid-filament plane of the extended sample. This region is monitored using laser microscopy, and the distance between the end-plates adjusted, thus obtaining a predefined stretch rate at the neck. To ensure a correct measurement, the sample should stay symmetric across the mid-filament plane as well as axisymmetric during extension. It is required that the gravitational sagging should be negligible. In some cases the rheological behavior of a polymer may in itself result in symmetry breaking [Matallah et al. (2006)] or axis-symmetric breaking [Rasmussen and Hassager (2001)] flow. In our experiments we have assured that our samples are axisymmetric and the mid-filament plane in the sample is the plane of symmetry at all time, based on the methodology from Bach et al. (2003a). For more details, see also Nielsen et al. (2008).

During the extension in a FSR, a load cell measures the elongational force, $F(t)$, and a laser micrometer measures the filament diameter, $2 R(t)$, at the mid-filament plane. The relevant strain in the elongation is the Hencky strain $(\epsilon)$, which is defined as $\epsilon(t)$ $=2 \ln \left(R_{0} / R(t)\right)$ for filament stretching of cylindrically shaped samples. $R_{0}$ is the midfilament radius and $L_{0}$ is the length of the sample at the start of the extension, at time

TABLE I. Linear viscoelastic parameters of the PI at $23^{\circ} \mathrm{C}$.

\begin{tabular}{ccccc}
\hline \hline$G_{N}^{0}$ & $n_{e}$ & $\tau_{\max }$ & $n_{g}$ & $\tau_{c}$ \\
\hline $470 \mathrm{kPa}$ & 0.25 & $75.6 \mathrm{~s}$ & 0.7 & $1.3 \mu \mathrm{s}$ \\
\hline \hline
\end{tabular}


$t=0$, as the sample may have been subjected to a pre-stretch. Initially, the sample is at rest for times $t<0$. The elongational strain rate or stretch rate is defined as $\dot{\epsilon}=d \epsilon / d t$.

With the assumption of axis-symmetric as well as a mid-filament symmetry plane, an expression for the difference between the axial and radial stress in the mid-filament plane was obtained by Szabo (1997). See also Szabo and McKinley (2003). In the absence of inertial and surface tension effects, the remaining terms can be written in the form

$$
\left\langle\sigma_{z z}-\sigma_{r r}\right\rangle+\frac{1}{2}\left\langle\sigma_{r r}-\sigma_{\theta \theta}\right\rangle+\frac{1}{2}\left\langle r \sigma_{r z}\right\rangle=\frac{F(t)-m_{f} g / 2}{\pi R(t)^{2}},
$$

where $F(t)$ is the axial force, $g$ is the gravitational acceleration, and $m_{f}$ is the weight of the polymer filament. The angular brackets, $\langle\ldots\rangle$, denote the average extensional stress at the mid-filament plane. If there are no rotation velocity of the sample (e.g., $v_{\theta}=0$ ) the second term on the left side in Eq. (5) is zero for Newtonian fluids. The second term is therefore also zero in the linear viscoelastic flow regime due to the correspondence principle of linear viscoelasticity [Pipkin (1972)]. McKinley and Sridhar (2002) stated that this argument may be extended to be applied for memory fluids.

It is a commonly used assumption that the stress and strain are uniform in the radial direction in the symmetry plane allowing the angular brackets in Eq. (5) to be omitted. This has been confirmed in a large number of computational studies by Kolte et al. (1997); Sizaire and Legat (1997); Hassager et al. (1998); Yao et al. (1998), Rasmussen and Hassager (1999, 2001); Yao et al. (2000); Bach et al. (2002); Sujatha et al. (2006); Matallah et al. (2007); Webster et al. (2008) and many more. These simulations have considered a wide variety of constitutive model and they all confirmed that deviations from uniform elongational flow are small at the mid-filament plane. They also confirmed that deviations from ideal elongational flow are small, particularly at higher Hencky strain values.

The third term on the left side of Eq. (5) may have an effect at small Hencky strain values, when a significant shearing component is present in the sample. At small aspect ratios $A_{\mathrm{i}}=L_{\mathrm{i}} / R_{\mathrm{i}}$, an extra shear contribution may add to the measured elongational force during the startup of the flow.

The effect of the additional shear may be reduced by a correction factor as discussed in details in the Appendix. The use of a correction factor requires an extension rate during pre-stretch considerably lower than the inverse of the largest relaxation time in the melt, as done in all our experiments. This is to ensure that stresses are build up in the linear viscoelasticity range only. In this case the correction factor gives the theoretical exact deviation from the ideal extensional stress at small strains, due to the correspondence principle of linear viscoelasticity [Pipkin (1972)]. At higher strains, where the use of a correction factor is less accurate, the deviations from ideal extension diminish.

Here the elongational stress in the sample at the mid-plane is then calculated as

$$
\sigma_{z z}-\sigma_{r r}=\frac{F(t)+m_{f} g / 2}{\pi R(t)^{2}} \cdot \frac{1}{1+\left(R(t) / R_{\mathrm{i}}\right)^{10 / 3} \cdot \exp \left(-A_{\mathrm{i}}^{3}\right) /\left(3 A_{\mathrm{i}}^{2}\right)}
$$

This formula is an approximation of the theoretically exact correction factor. It theoretically ensures that the initial true elongational stress will stay within $3 \%$ of this corrected stress if $A_{\mathrm{i}} \geq 0.3$. The initial correction factor is about $13 \%$ in all our experiments and becomes negligible at higher strains, as $R_{i}=2.5 \mathrm{~mm}, L_{i}=1.5 \mathrm{~mm}$, and $R_{0}$ is about $1.5 \mathrm{~mm}$. 


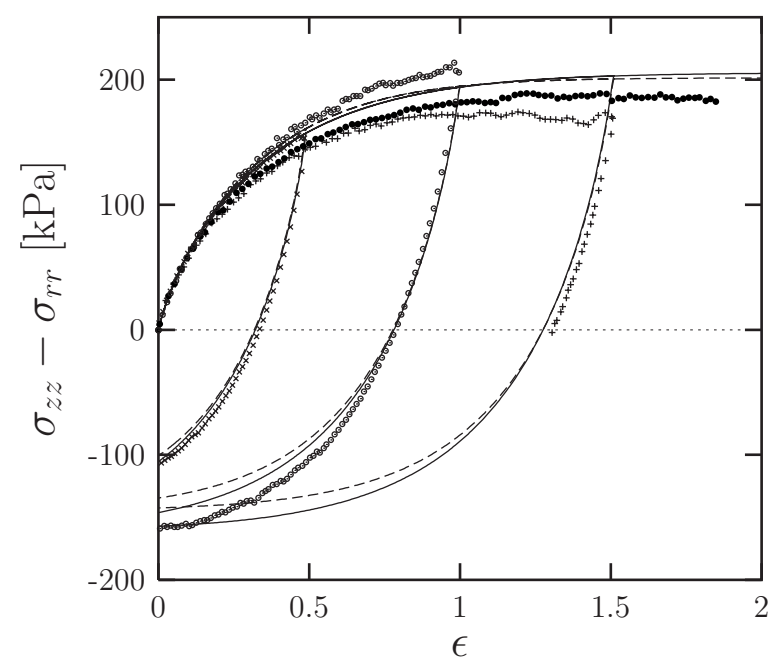

FIG. 2. Elongational stress as a function of the Hencky strain at $23{ }^{\circ} \mathrm{C}$. Startup and reversing elongation of the PI with $|\dot{\epsilon}|=0.01 \mathrm{~s}^{-1}$ corresponding to a De number of $\mathrm{De}=0.42$. The solid lines (-) are predictions from Eq. (1) corresponding to the measurements. The dashed lines (-- ) are predictions from Eq. (2) corresponding to the measurements.

\section{REVERSED FLOW}

The transient uni-axial extension is up to a Hencky strain of $\epsilon_{0}$. This uni-axial stretching is followed by a transient bi-axial squeeze. During the startup of uni-axial stretching, we define the rate as $\dot{\epsilon}^{+}=\dot{\epsilon}$ and in the bi-axial squeeze the rate is defined as $\dot{\epsilon}^{-}=-\dot{\epsilon}$. Both $\dot{\epsilon}^{+}$and $\dot{\epsilon}^{-}$will stay positive, constant, and equal $\left(\dot{\epsilon}^{+}=\dot{\epsilon}^{-}\right)$during the extension.

As a commonly used characteristic relaxation time for the polymers, we define

$$
\tau_{a}=\int_{0}^{\infty} G(s) s d s / \int_{0}^{\infty} G(s) d s \approx \tau_{\max }\left(\frac{1+n_{e}}{2+n_{e}}\right)=42 \mathrm{~s} .
$$

More details can be found in Bach et al. (2002) as well as Bach et al. (2003a). This definition is within $5 \%$ of the Doi-Edwards reptation time. We use this relaxation time to define a Deborah number for the elongational flow, $\mathrm{De}=\tau_{a} \cdot \dot{\epsilon}$.

Figure 2 shows the elongational stress as a function of the Hencky strain until $\epsilon_{0}$ where the flow is reversed. The extension rates are all $0.01 \mathrm{~s}^{-1}$ giving a fixed De of 0.42 . The stress saturates at a strain of about unity at this Deborah number due to the relaxation of the molecule. At low values of $\epsilon_{0}$, it is possible to reverse the flow completely. One has to keep in mind as the strain goes toward zero during the reversed flow the data are still corrected with Eq. (6), with an applied correction factor of maximal $13 \%$. This may not be the exact theoretically procedure to apply. At higher $\epsilon_{0}$ values, the sample buckles due to the compression force and a reliable stress cannot be measured for negative stresses. At even higher $\epsilon_{0}$ values, a crack is observed in the surface which gradually increases in size, sometime followed by a complete rupture. This behavior removes the possibility to measure the stress at higher strain values. Measurements with a crack have not been presented in this paper. Figure 3 shows the stress variation as a function of the Hencky strain at higher Deborah number with a maximal value of $\mathrm{De}=4.2$, representing a highly elastic deformation. $\epsilon_{0}$ are all unity in this figure. The theoretical predictions found using Eq. (1) (solid lines) and Eq. (2) (dashed lines) show an agreement which is within the 


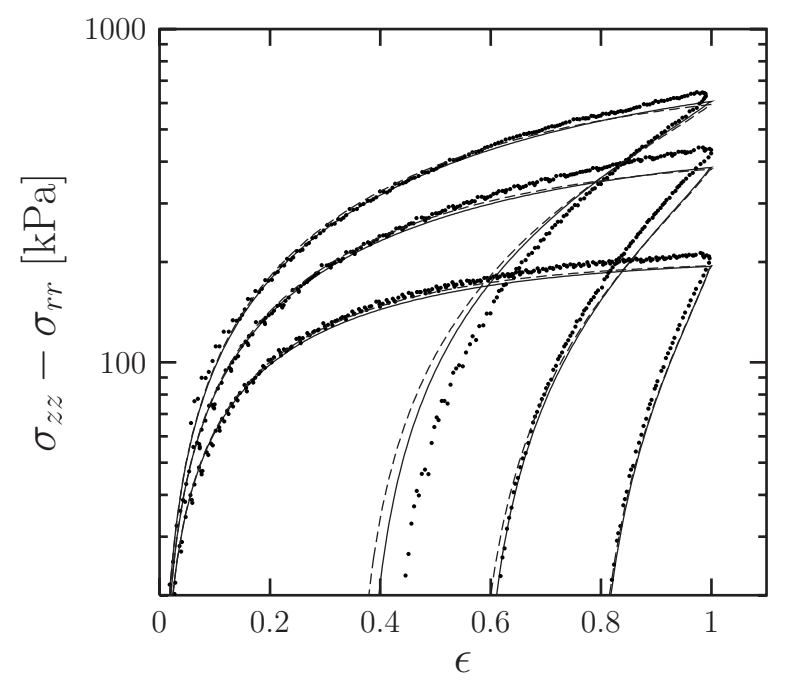

FIG. 3. Elongational stress as a function of the Hencky strain at $23^{\circ} \mathrm{C}$. Startup and reversing elongation of the PI with $|\dot{\epsilon}|=0.01 \mathrm{~s}^{-1}$ (bottom), $|\dot{\epsilon}|=0.03 \mathrm{~s}^{-1}$ (middle), and $|\dot{\epsilon}|=0.1 \mathrm{~s}^{-1}$ (top). The corresponding De numbers are $\mathrm{De}=0.42$ (bottom), $\mathrm{De}=1.26$ (middle), and $\mathrm{De}=4.2$ (top). The solid lines (一) are predictions from Eq. (1), the dashed lines $(---)$ are predictions from Eq. (2), and the dots $(-)$ are the corresponding measurements.

accuracy of the measurements in both figures. Notice that the extension data, in the linear viscoelasticity regime at low Hencky strains, in both Fig. 2 and 3 agree within 10\% with the linear viscoelasticity based on the shear flow (the lines).

In elongational flow, the Doi and Edwards strain tensor model with [Eq. (1)] or without [Eq. (2)], the independent alignment approximation predicts an upper limit to the extensional stress of 5 or $15 / 4$ times the plateau modulus $\left(G_{N}^{0}\right)$, respectively. These values are only about three times higher than our maximal measured extensional stress.

To include all our measurements in one figure (Fig. 4), the "strain recovery" is shown as a function of the maximal imposed Hencky strain where the flow is reversed $\epsilon_{0}$. The classical measurement of reversed elongational flow is the elastic recovery. Here the flow is reversed flow by removing externally applied forces on the sample. The strain history is unknown during the reversed flow. Both the stress and the strain history are known for a strain recovery experiment. The strain recovery or $\epsilon_{R}$ is defined to be the strain at which the stress in the filament changes sign from positive to negative stress, relative to the maximal imposed strain. In mathematical terms this implies $\epsilon_{R}=\epsilon_{0}-\epsilon\left(t_{R}\right)$ where $t_{R}$ is the time at which the stress changes sign from positive to negative value, e.g., $\sigma_{11}-\sigma_{33}=0$. It is the necessary bi-axial strain to reach zero extensional stress. The experimental as well as theoretical procedure to determine the strain recovery can be found in Nielsen and Rasmussen (2008). The dotted line where $\epsilon_{R}=\epsilon_{0}$ represents a complete elastic retraction of the sample which is the theoretical maximal value of $\epsilon_{R}$ in Fig. 4. At low De numbers, $\epsilon_{R}$ saturates, whereas at $\mathrm{De}=4.5$, the strain recovery continues to increase with values much closer to a complete elastic retraction of the sample. The maximal experimentally obtainable $\epsilon_{0}$ is limited by the initiation of the sample rupture. The theoretical prediction of the strain recovery with the assumption of pure configurational stress using Eqs. (1) and (2) is within the accuracy of the measurements.

Outside this pure configurational stress regime, it is common to multiply a stretch evolution function on the pure configurational stress. The values of the strain recovery are independent of this stretch evolution function. As showed experimentally by Nielsen and 


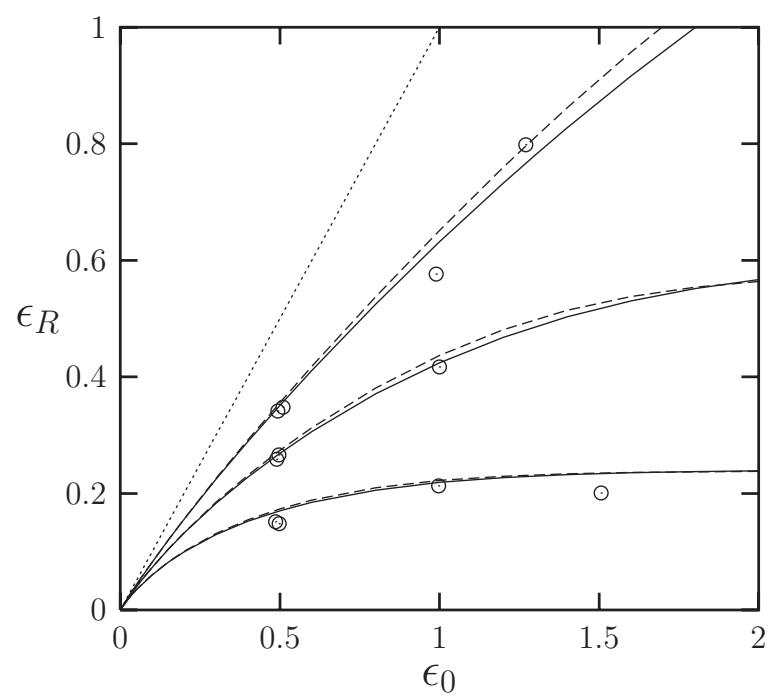

FIG. 4. Recovery strain value, $\epsilon_{R}$, plotted as against the maximal Hencky strain, $\epsilon_{0}$. The solid lines (-) are predictions from Eq. (1), the dashed lines $(---)$ are predictions from Eq. (2), and the circles $(\bigcirc)$ are the corresponding measurements, at $\mathrm{De}=0.42$ (bottom), $\mathrm{De}=1.26$ (middle), and $\mathrm{De}=4.2$ (top).

Rasmussen (2008) and Rasmussen et al. (2009), using the strain recovery, is this theoretical extension of the pure configurational stress theory not valid. These experiments were based on linear and branched narrow molecular mass distribution polystyrene, respectively.

\section{CONCLUSION}

The filament stretching rheometer was used for measuring the startup of uni-axial elongational flow followed by reversed bi-axial flow in the non-linear flow regime on an almost monodisperse polyisoprene. The time of the flow during extension was considerably smaller than the Rouse time of the polyisoprene. The pure configurational stress assumption, both used here in the independent alignment as well as in the entangled network form, predicts stresses within the scattering of the presented data. This allow us to conclude that the assumption of pure configurational stresses relaxed by reptation to describe the flow of polymers melts and entangled liquids do not need any revision.

\section{ACKNOWLEDGMENT}

The authors gratefully acknowledge financial support from the Danish Technical Research Council.

\section{APPENDIX}

At small aspect ratios $\left(A_{i}=L_{i} / R_{i}\right)$, an extra shear contribution may add to the measured elongational force during the startup of the flow in the FSR. The effect of this shear stress may be eliminated with the use of a correction factor. The original and-until now-only existing correction factor was suggested by Spiegelberg et al. (1996) as

$$
\sigma_{z z}-\sigma_{r r}=\frac{F(t)+m_{f} g / 2}{\pi R(t)^{2}} \cdot \frac{1}{1+\left(R(t) / R_{i}\right)^{14 / 3} /\left(3 A_{i}^{2}\right)} .
$$




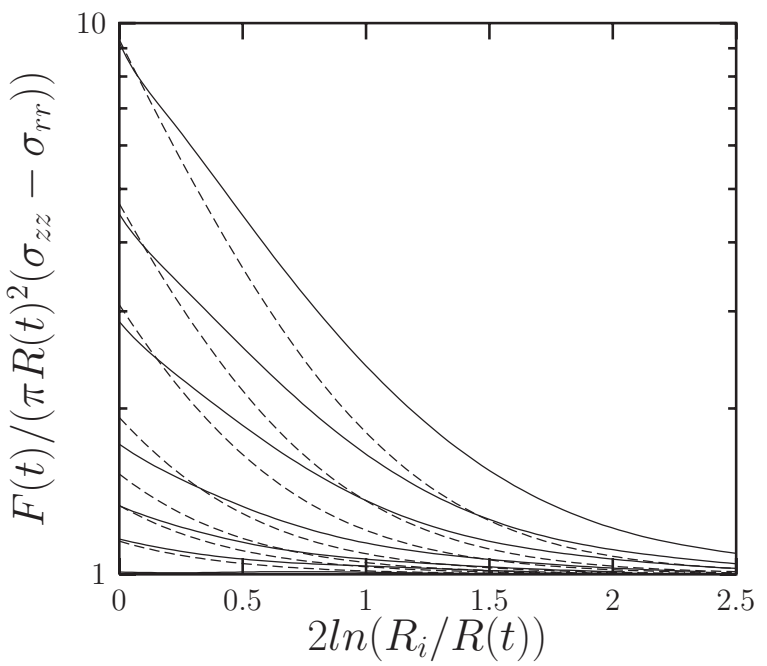

FIG. 5. The correction factor [e.g., $F(t) /\left(\pi R(t)^{2}\left(\sigma_{z z}-\sigma_{r r}\right)\right)$ ] as a function of the imposed pre-stretch. The solid lines (-) are the numerical calculated correction factor. The dashed lines (- - ) are predictions from Eq. (A1). The initial aspect ratios are $A_{\mathrm{i}}=L_{\mathrm{i}} / R_{\mathrm{i}}$ are $0.2,0.3,0.4,0.6,0.8,1.0,1.5$ from the top to bottom line, respectively.

The correction factor in Eq. (A1) is written here in a potentially pre-stretched configuration. This formula (A1) is based on a lubrication analysis and is analytical correct for small strains and sample aspect ratios for all types of fluids.

We have compared this formula (A1) with corresponding numerical computations of the correction factor [e.g., $F(t) /\left(\pi R(t)^{2}\left(\sigma_{z z}-\sigma_{r r}\right)\right)$ ] for a Newtonian fluid in Fig. 5. $\sigma_{z z}$ $-\sigma_{r r}$ is the ideal extensional stress. The numerical computations are the accurate values to use as correction factors. The correction factor formula by Spiegelberg et al. (1996)

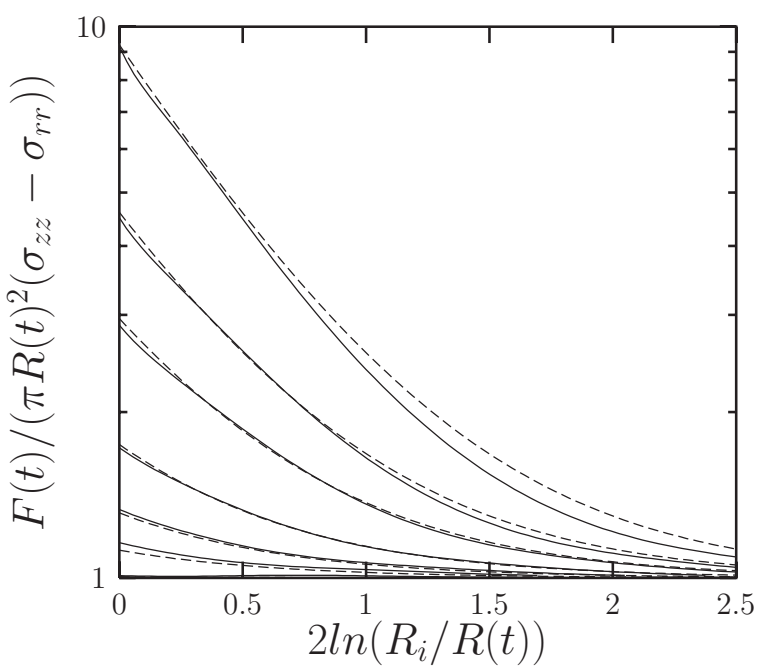

FIG. 6. The correction factor [e.g., $F(t) /\left(\pi R(t)^{2}\left(\sigma_{z z}-\sigma_{r r}\right)\right)$ ] as a function of the imposed pre-stretch. The solid lines (-) are the numerical calculated correction factor. The dashed lines (- - ) are predictions from Eq. (6). The initial aspect ratios are $A_{\mathrm{i}}=L_{\mathrm{i}} / R_{\mathrm{i}}$ are $0.2,0.3,0.4,0.6,0.8,1.0,1.5$ from the top to bottom line, respectively. 
can give as much as $20 \%$ deviation from the numerical values. Due to the correspondence principle of linear viscoelasticity [Pipkin (1972)], these numerical calculated values will be exactly the same in the linear viscoelastic range for all types of fluids. Details of the applied numerical method and the performing of the computations can be found in Rasmussen (2000) and Rasmussen and Hassager (1999), respectively.

In Fig. 6 we have compared the our empirically based formula (6) with the corresponding numerical computations of the correction factor [e.g., $F(t) /\left(\pi R(t)^{2}\left(\sigma_{z z}-\sigma_{r r}\right)\right)$ ] for a Newtonian fluid, similar to Fig. 5. The correction factor given by formula (6) will give less than $3 \%$ deviation from the numerical calculated values if $A_{\mathrm{i}} \geq 0.3$.

\section{References}

Adams, J. M., and P. D. Olmsted, "Nonmonotonic models are not necessary to obtain shear banding phenomena in entangled polymer solutions," Phys. Rev. Lett. 102, 067801 (2009).

Auhl, D., J. Ramirez, A. E. Likhtman, P. Chambon, and C. Fernyhough, "Linear and nonlinear shear flow behavior of monodisperse polyisoprene melts with a large range of molecular weights," J. Rheol. 52, 801-835 (2008).

Bach, A., K. Almdal, H. K. Rasmussen, and O. Hassager, "Elongational viscosity of narrow molar mass distribution polystyrene," Macromolecules 36, 5174-5179 (2003a).

Bach, A., H. K. Rasmussen, and O. Hassager, "Extensional viscosity for polymer melts measured in the filament stretching rheometer," J. Rheol. 47, 429-441 (2003b).

Bach, A., H. K. Rasmussen, P. Y. Longin, and O. Hassager, "Growth of non-axisymmetric disturbances of the free surface in the filament stretching rheometer: Experiments and simulation," J. Non-Newtonian Fluid Mech. 108, 163-186 (2002).

Baumgaertel, M., A. Schausberger, and H. H. Winter, "The relaxation of polymers with linear flexible chains of uniform length,” Rheol. Acta 29, 400-408 (1990).

de Gennes, P. G., Scaling Concepts in Polymer Physics (Cornell University Press, Ithaca, NY, 1979).

Dhole, S., A. Leygue, C. Bailly, and R. Keunings, "A single segment differential tube model with interchain tube pressure effect," J. Non-Newtonian Fluid Mech. 161, 10-18 (2009).

Doi, M., and S. F. Edwards, "Dynamics of concentrated polymer systems. Part 3.-The constitutive equation," J. Chem. Soc., Faraday Trans. 2 74, 1818-1832 (1978).

Doi, M., and S. F. Edwards, The Theory of Polymer Dynamics (Clarendon, Oxford, UK, 1986).

Fang, J., M. Kröger, and H. C. Öttinger, "A thermodynamically admissible reptation model for fast flows of entangled polymers. II. Model predictions for shear and extensional flows,” J. Rheol. 44, 1293-1317 (2000).

Hassager, O., M. I. Kolte, and M. Renardy, "Failure and nonfailure of fluid filaments in extension," J. NonNewtonian Fluid Mech. 76, 137-151 (1998).

Ianniruberto, G., and G. Marrucci, "A simple constitutive equation for entangled polymers with chain stretch," J. Rheol. 45, 1305-1318 (2001).

Joshi, Y. M., and M. M. Denn, "Rupture of entangled polymeric liquids in elongational flow," J. Rheol. 47, 291-298 (2003).

Khaliullin, R. N., and J. D. Schieber, "Self-consistent modeling of constraint release in a single-chain meanfield slip-link model," Macromolecules 42, 7504-7517 (2009).

Kolte, M. I., H. K. Rasmussen, and O. Hassager, "Transient filament stretching rheometer II: Numerical simulation," Rheol. Acta 36, 285-302 (1997).

Likhtman, A., "Single-chain slip-link model of entangled polymers: Simultaneous description of neutron spinecho, rheology, and diffusion," Macromolecules 38, 6128-6139 (2005).

Lyhne, A., H. K. Rasmussen, and O. Hassager, "Simulation of elastic rupture in extension of entangled monodisperse polymer melts," Phys. Rev. Lett. 102, 138301 (2009).

Marrucci, G., and N. Grizzuti, "Fast flows of concentrated polymers-Predictions of the tube model on chain 
stretching," Gazz. Chim. Ital. 118, 179-185 (1988).

Marrucci, G., and G. Ianniruberto, "Interchain pressure effect in extensional flows of entangled polymer melts," Macromolecules 37, 3934-3942 (2004).

Matallah, H., M. J. Banaai, K. S. Sujatha, and M. F. Webster, "Modelling filament stretching flows with strain-hardening models and sub-cell approximations," J. Non-Newtonian Fluid Mech. 134, 77-104 (2006).

Matallah, H., K. S. Sujatha, M. J. Banaai, and M. F. Webster, "Single and multi-mode modelling for filament stretching flows," J. Non-Newtonian Fluid Mech. 146, 92-113 (2007).

McKinley, G. H., and T. Sridhar, "Filament-stretching rheometry of complex fluids," Annu. Rev. Fluid Mech. 34, 375-415 (2002).

McLeish, T. C. B., and R. G. Larson, "Molecular constitutive equations for a class of branched polymers: The pom-pom polymer," J. Rheol. 42, 81-110 (1998).

Mead, D. W., R. G. Larson, and M. Doi, “A molecular theory for fast flows of entangled polymers," Macromolecules 31, 7895-7914 (1998).

Milner, S. T., and T. C. B. McLeish, "Reptation and contour-length fluctuations in melts of linear polymers," Phys. Rev. Lett. 81, 725-728 (1998).

Nielsen, J. K., and H. K. Rasmussen, "Reversed extension flow," J. Non-Newtonian Fluid Mech. 155, 15-19 (2008).

Nielsen, J. K., H. K. Rasmussen, and O. Hassager, "Stress relaxation of narrow molar mass distribution polystyrene following uni-axial extension,” J. Rheol. 52, 885-899 (2008).

Pearson, D. S., A. D. Kiss, L. J. Fetters, and M. Doi, "Flow-induced birefringence of concentrated polyisoprene solutions," J. Rheol. 33, 517-535 (1999).

Pipkin, A. C., Lectures in Viscoelasticity Theory (Springer, New York, 1972).

Rasmussen, H. K., "Lagrangian viscoelastic flow computations using the Rivlin-Sawyers constitutive model," J. Non-Newtonian Fluid Mech. 92, 227-243 (2000).

Rasmussen, H. K., J. H. Christensen, and S. J. Gottsche, "Inflation of polymer melts into elliptic and circular cylinders," J. Non-Newtonian Fluid Mech. 93, 245-263 (2000).

Rasmussen, H. K., and O. Hassager, "Three-dimensional simulations of viscoelastic instability in polymeric filaments," J. Non-Newtonian Fluid Mech. 82, 189-202 (1999).

Rasmussen, H. K., and O. Hassager, "The role of surface tension on the elastic decohesion of polymeric filaments," J. Rheol. 45, 527-537 (2001).

Rasmussen, H. K., P. Laille, and K. Yu, "Large amplitude oscillatory elongation flow," Rheol. Acta 47, 97-103 (2008).

Rasmussen, H. K., A. L. Skov, J. K. Nielsen, and P. Laille, "Elongational dynamics of multiarm polystyrene," J. Rheol. 53, 401-415 (2009).

Schieber, J., J. Neergaard, and S. Gupta, "A full-chain, temporary network model with sliplinks, chain-length fluctuations, chain connectivity and chain stretching," J. Rheol. 47, 213-233 (2003).

Sizaire, R., and V. Legat, "Element simulation of a filament stretching extensional rheometer," J. NonNewtonian Fluid Mech. 71, 89-107 (1997).

Spiegelberg, S. H., D. C. Ables, and G. H. McKinley, "The role of end-effects on measurements of extensional viscosity in filament stretching rheometers," J. Non-Newtonian Fluid Mech. 64, 229 (1996).

Sujatha, K. S., H. Matallah, M. J. Banaai, and M. F. Webster, "Computational predictions for viscoelastic filament stretching flows: ALE methods and freesurface techniques (CM and VOF)," J. Non-Newtonian Fluid Mech. 137, 81-102 (2006).

Szabo, P., "Transient filament stretching rheometer part I: Force balance analysis," Rheol. Acta 36, 277-284 (1997).

Szabo, P., and G. H. McKinley, "Filament stretching rheometer: Inertia compensation revisited," Rheol. Acta 42, 269-271 (2003).

Urakawa, O., M. Takahashi, T. Masuda, and N. Golshan Ebrahimi, "Damping functions and chain relaxation in uniaxial and biaxial extensions: Comparison with the Doi-Edwards theory," Macromolecules 28, 71967201 (1995).

Wagner, M. H., S. Kheirandish, and O. Hassager, "Quantitative prediction of the transient and steady-state elongational viscosity of nearly monodisperse polystyrene melts," J. Rheol. 49, 1317-1327 (2005). 
Wagner, M. H., V. H. Rolon-Garrido, J. K. Nielsen, H. K. Rasmussen, and O. Hassager, "A constitutive analysis of transient and steady-state elongational viscosities of bidisperse polystyrene blends," J. Rheol. 52, 67-86 (2008).

Webster, M. F., H. Matallah, K. S. Sujatha, and M. J. Banaai, "Numerical modelling of step-strain for stretched filaments," J. Non-Newtonian Fluid Mech. 151, 38-58 (2008).

Yao, M. W., G. H. McKinley, and B. Debbaut, "Extensional deformation, stress relaxation and necking failure of viscoelastic filaments," J. Non-Newtonian Fluid Mech. 79, 469-501 (1998).

Yao, M. W., S. H. Spiegelberg, and G. H. McKinley, "Dynamics of weakly strain-hardening fluids in filament stretching devices," J. Non-Newtonian Fluid Mech. 89, 1-43 (2000). 